\title{
Data Movement Optimizations for Independent MPI I/O on the Blue Gene/Q
}

\author{
Preeti Malakar*, Venkatram Vishwanath* \\ Argonne National Laboratory
}

\begin{abstract}
Scalable high-performance I/O is crucial for application performance on large-scale systems. With the growing complexity of the system interconnects, it has become important to consider the impact of network contention on I/O performance because the I/O messages traverse several hops in the interconnect before reaching the I/O nodes or the file system. In this work, we present a route-aware and load-aware algorithm to modify existing bridge node assignment in the Blue Gene/Q (BG/Q) supercomputer. We reduce the network contention and reduce the write time by an average of $60 \%$ over the default independent $\mathrm{I} / \mathrm{O}$ and by $20 \%$ over collective I/O on up to 8192 nodes on the Mira BG/Q system. Our algorithm routes $1.4 \times$ fewer messages through the bridge nodes which connect to the $\mathrm{I} / \mathrm{O}$ nodes on the BG/Q. Our algorithm also reduces the average distance of a compute node from a bridge node, and thus lessens the network load, and decreases I/O time.
\end{abstract}

Keywords: Independent I/O, Data movement, Intra-node data aggregation, Interconnect routing, File write

\section{Introduction}

Parallel applications need to store persistent data for a myriad of purposes - checkpoint, visualization, analysis etc. Therefore, the I/O subsystem is a crucial part of any cluster or supercomputer installation. Numerous efforts exist to optimize parallel I/O like data sieving and collective I/O optimizations [21, 22, 30, 33] and sophisticated distributed locking in parallel file systems such as GPFS [28]. I/O nodes or I/O servers have been introduced for I/O forwarding and I/O isolation $[15,17]$. However, there has been less focus on the system interconnect through which the I/O messages are routed. With the advent of complex interconnects like dragon-fly and high-dimension torus [12], the messages being written out to the filesystem first traverse through the system interconnect to the I/O nodes en-route to the filesystem. The inverse order holds true for messages/data being read. I/O messages are routed through multiple hops in the interconnect which can cause congestion within the interconnect [15]. In this work, we advocate the importance of considering the interconnect for high-performance I/O. We need to carefully analyze any I/O bottlenecks present within the complex interconnects in order to further enhance the I/O performance.

\footnotetext{
${ }^{*}$ Corresponding author

Email addresses: pmalakar@anl.gov (Preeti Malakar), venkat@anl.gov (Venkatram Vishwanath)
} 


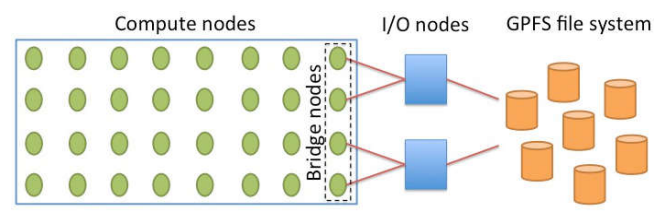

Figure 1: Bridge nodes in BG/Q connect to the $\mathrm{I} / \mathrm{O}$ nodes, which connect to the file system.

Applications running on high-performance systems mainly use the optimized MPI-IO [30] for parallel reads and writes to the file system or use high-level libraries such as HDF5 which in turn use MPI-IO. MPI-IO has two flavors - independent I/O and collective I/O. On some platforms, collective I/O outperforms independent I/O [21, 22] due to the several optimizations in collective $\mathrm{I} / \mathrm{O}$ related to file system access. In some cases, independent I/O may perform better than collective I/O [24, 25]. Many applications and benchmarks including S3D [13], FLASH [21], HACC [2], Energy2 [9], Turbulence3 [9], MADBench2 [8], HOMME [8] etc. have the option of using independent I/O [10]. The performance of independent vs. collective I/O for future burst buffers and NVRAM [16, 23] is not yet well studied.

In this work, we focus on independent I/O on Blue Gene/Q and show the efficacy of our algorithm over both collective and the default independent I/O. We have used the Mira supercomputer at Argonne National Laboratory. Mira is an IBM Blue Gene/Q system with 786,432 cores $(49,152$ nodes $)$ and a $5 \mathrm{D}$ torus interconnect. A subset of the compute nodes, called bridge nodes [12,19] are the gateway nodes for I/O traffic. Figure 1 shows a schematic of the BG/Q compute node and I/O node architecture. Each compute node has 10 links for communication within the torus, each link of $2 \mathrm{~GB} / \mathrm{s}$ bandwidth. Every compute node is assigned a bridge node. The bridge nodes connect to $\mathrm{I} / \mathrm{O}$ nodes over an $11^{\text {th }}$ serial $\mathrm{I} / \mathrm{O}$ link at $2 \mathrm{~GB} / \mathrm{s}$. 2 bridge nodes connect to one I/O node. I/O nodes perform I/O on behalf of the compute nodes. Mira is configured with one I/O node per 128 compute nodes for I/O isolation [5].

The bridge nodes are selected during partition boot time depending on the partition in which the job is running. They are attached to an already booted I/O block [11, 17]. The deterministic/static routing order and the torus connectivity of the partition determine the paths taken by the I/O messages from a compute node to its bridge node [12]. The default path taken by the I/O messages in a machine like BG/Q with a complex interconnect topology does not ensure that the messages travel on paths with minimum congestion. We examine the route taken by I/O messages from a compute node to its bridge node for independent I/O using the BG/Q routing algorithm. We show the I/O paths from compute nodes which were assigned bridge node 98 on Mira in Figure 2 for a 512-node job with 1 rank per node. The edge width indicates the number of messages being routed through that edge. It can be clearly seen that some paths are very heavily loaded as compared to others. This leads to congestion and hence poor I/O performance. The load will be higher for jobs with multiple ranks per node because multiple messages from a node will traverse on the same network links from a source to its destination due to static routing. In the default case, a bridge node may be present in the path from a compute node to its destination bridge node because assignments are not always to the closest bridge nodes. This results in more congested links. 


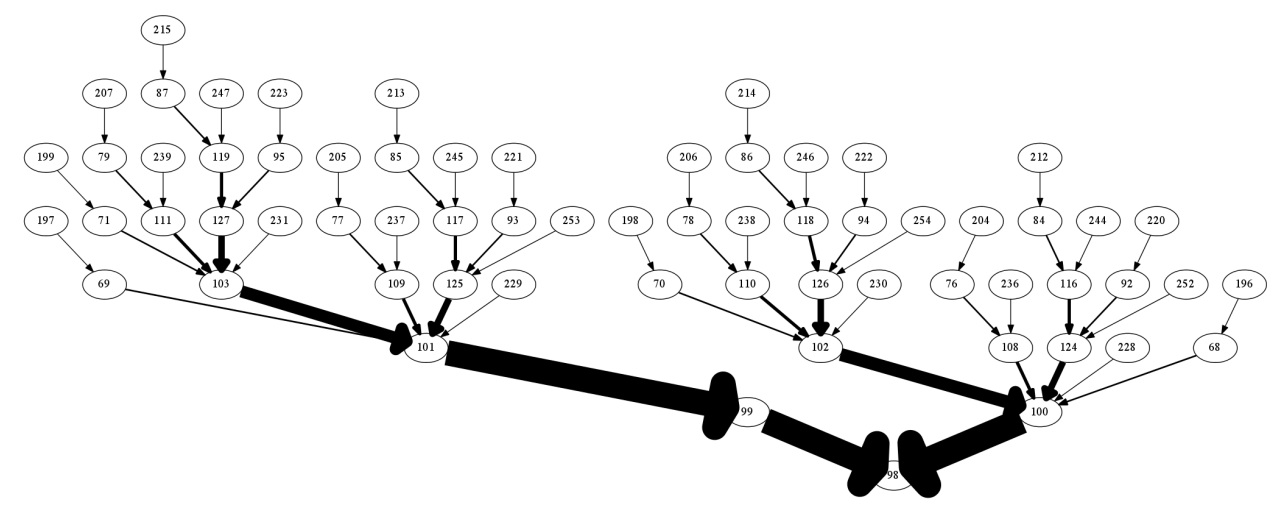

Figure 2: Path for I/O messages from the compute nodes, whose assigned bridge node is node 98 on Mira. Few of the network links have very high load. Maximum load in this network is 32 , on the edge $100 \rightarrow 98$.

In this paper, we present an algorithm for optimizing independent MPI-IO by reducing the network congestion. The default algorithm for determining a bridge node for a compute node assigns equal number of compute nodes per bridge node. Though this results in same number of I/O requests per bridge node, yet the physical network links have an uneven distribution of load (packets traveling on the link) due to deterministic routing. Furthermore, for some compute nodes, the distance (hops) to their respective bridge nodes is large due to inefficient bridge node assignments. This also results in a few highly congested links, due to multiple messages routed on a link, as shown in Figure 2. This increases the time taken by I/O messages to reach the bridge nodes and hence causes I/O bottleneck. Though BG/Q has a low-latency high-bandwidth network, yet longer hops on congested links to the bridge nodes result in latency-bound I/O in some cases. We have developed an algorithm that reduces the average distance of a compute node from a bridge node, and thus lessens the network load, and decreases I/O time.

Our approach is to determine a different bridge node for some of the compute nodes. The objective is to find a bridge node that is closer to a compute node than its default bridge node. The closer a bridge node is to a compute node, the fewer the average distance traveled by I/O messages and lesser the overall network contention. We solve this problem by building a connectivity tree for each bridge node, and then traversing each tree using the global knowledge from all other trees. We not only assign a closer bridge node but also ensure that the number of new assignments per bridge node is similar, so that the load (number of I/O requests) is still balanced across all bridge nodes as in the default case.

The unbalanced network load results in huge variation in the $\mathrm{I} / \mathrm{O}$ completion times per process. The time taken by 4096 processes to write a 4 GB file to the I/O nodes varies between $0.13-0.72 \mathrm{sec}$ across the different processes using independent MPI-IO. Measuring the time to write to the $\mathrm{I} / \mathrm{O}$ nodes helps understand interconnect-related bottleneck in isolation from file system-related performance bottlenecks. This huge variation is not only due to longer hops but also due to the routing order which results in many I/O messages routed along the same links. In our algorithm, we reduce this congestion by considering the network route taken by I/O messages while determining the new assignments. The network tree for a bridge node is built such that the path from any node in the tree to the bridge 
node is its default path to the bridge node. A default path is the path determined by the static routing order of $\mathrm{BG} / \mathrm{Q}$. Also, the paths in a tree are distinct from paths in other trees. Thus, when we assign a new bridge node to the nodes in its tree, the paths of I/O messages from these nodes to the bridge node are distinct from paths taken by nodes in other trees corresponding to other bridge nodes. Hence, there is less interference on a path in a tree from traffic to other bridge nodes and less overlap of messages on same links for the new assignments.

The overhead in our approach is the explicit send/receive of I/O data because the compute nodes are assigned a bridge node at boot time, which cannot be changed during runtime from user-space application. Despite this, our load-aware and route-aware algorithm improves independent I/O time by mitigating the bottlenecks in the network. We combine messages from multiple ranks in a node and send to the bridge nodes. Using our algorithm, with and without this coalescing technique, we not only improve write times to the file system over the default independent I/O, but also improve the write time to the bridge nodes over both independent and collective I/O. This will be very useful in future systems equipped with burst buffers which may be connected to few gateway nodes in the system [23], similar to the current I/O node architecture.

We study the impact of our algorithm on smaller and larger writes, ranging from $8 \mathrm{~KB}-$ $4 \mathrm{MB}$ writes per MPI rank using independent MPI-IO. It is known that smaller independent writes have worse performance [10]. However, our results show an average improvement of $60 \%$ over the default independent MPI-IO on 512 - 131072 processes on Mira. Our optimized independent $\mathrm{I} / \mathrm{O}$ also improves performance by an average of $20 \%$ over collective $\mathrm{I} / \mathrm{O}$ while writing to the I/O nodes, data size ranging from $128 \mathrm{~KB}-8 \mathrm{MB}$ per MPI rank from 8192 - 131072 MPI processes. Our results also show that the default independent MPI-IO on Mira routes $1.4 \times$ more I/O messages through the bridge nodes as a result of inefficient bridge node assignments. With intra-node aggregation, we achieved $10.5 \mathrm{~GB} / \mathrm{s}$ write bandwidth at 4096 nodes writing $64 \mathrm{~GB}$ file, as compared to $0.7 \mathrm{~GB} / \mathrm{s}$ with default MPI independent I/O.

The main contributions of our work are summarized below:

- We present an algorithm to find better bridge node assignments for compute nodes via better routing and shorter hops to the bridge nodes.

- We built a connectivity tree for each bridge node and confine the I/O traffic to a bridge node to its tree edges so that there is reduced network congestion.

- We aggregate data at one or more cores on the node before sending to the respective bridge node cores, this led to lesser network congestion and coalesced accesses.

- Our algorithm is able to route the $\mathrm{I} / \mathrm{O}$ messages more efficiently than the default algorithm in BG/Q. Our algorithm gives $60 \%$ better performance on average than the default MPI independent I/O.

\section{Related Work}

There has been a lot of work on I/O optimization, most of the efforts being for collective writes [14, 30, 32]. Chen et al. [14] propose physical data layout-aware rearranging and reordering of MPI-IO accesses. Wang et al. [32] propose reorganizing I/O requests within 
each file domain to reduce contention at the file system. These efforts optimize accesses to file systems which are optimized for aligned large-chunk block-level accesses [28, 29]. In this work, we focus on path of I/O messages in the interconnect, through which all I/O messages are routed in modern supercomputers.

Dillow et al. proposed nearest neighbour, round robin and projection routing configurations for avoiding network congestion in Cray systems at OLCF [15]. They improved the I/O bandwidth by modifying the pairing of clients to storage servers. While they were able to alter the I/O server and router configurations, we had no such option in BG/Q. We improve the $\mathrm{I} / \mathrm{O}$ performance from user-space for a given $\mathrm{I} / \mathrm{O}$ configuration which is determined by the job's allocated partition.

Bhatele et al. [7] study the affect of allocated partition and interference due to other jobs on a job's performance. They assert that resource contention due to other jobs can introduce performance variability in $\mathrm{I} / \mathrm{O}$. We found that this variability is also attributed to inefficient routing of I/O messages to the bridge nodes. We improve the routing of I/O messages in the network of the allocated partition and improve the I/O performance as well as decrease the variability in write times. Hence our work will also impact the overall throughput of jobs. Uselton et al. [31] examine I/O variability using statistical analysis to identify root causes of performance degradation. Our work can benefit from such analysis which can reveal more information on the role of interconnect on $\mathrm{I} / \mathrm{O}$ performance.

Previous studies $[6,20]$ highlight contention in torus networks of Blue Gene systems in the context of MPI communication, we optimize the contention in the context of I/O. Rajachandrasekar et al. [27] propose a QoS-aware data staging framework to isolate I/O traffic, because the system network is generally space-shared between MPI communication and file system traffic. Our algorithm improves I/O time by better routing the I/O traffic within the network. Hence our approach can further improve I/O time when traffic is isolated using QoS capabilities of a network.

\section{Route-aware and Load-aware Algorithm for Independent I/O}

In this section, we present our algorithm for optimized independent MPI I/O. In the default case of independent MPI I/O on Blue Gene/Q, all the MPI processes running on the compute nodes write their data through the default bridge nodes (a small subset of compute nodes). Every compute node has a statically assigned default bridge node for the duration of the job runtime. The bridge nodes are assigned at the boot time of the compute node partition(s). These bridge nodes are connected to the I/O nodes via the $11^{\text {th }}$ link and hence all the data to be written to the filesystem from the compute nodes is routed through the bridge nodes [11]. Two bridge nodes are connected to an I/O node. Every bridge node has an equal number of compute nodes assigned to them. However, the default algorithm of this compute node-to-bridge node assignment results in longer hops to the default bridge node for several compute nodes. This leads to longer paths for the I/O messages within the BG/Q interconnect. This causes congestion and a significant load imbalance on the physical network links as shown in Figure 2. Thus it increases I/O times despite high-bandwidth interconnect.

We resolve the network congestion problem for I/O by constructing subtrees for each bridge node and confine most of the I/O traffic to these subtrees. We construct these trees using the knowledge about the BG/Q routing along the tree edges, so that the nodes of a tree send data to its root node on the edges of this tree. Our approach is to assign a different 
bridge node for some of the compute nodes based on the distance from the new bridge node. This is programmatically achieved in user-space. If a new bridge node can be found which is fewer hops away than the default bridge node, we consider the possibility of assigning this new bridge node to the compute node. However, we are cautious in modifying the assignment of every compute node due to the overhead involved with new assignments. The overhead is due to the fact that the reassignment entails explicit sending of I/O messages to the newly assigned bridge node. In the default case, the bridge node is already known at boot time and hence the MPI write call initiates the implicit sending of I/O messages to the default bridge node. Whereas, in our case, the compute nodes with new bridge nodes have to explicitly send the I/O data via MPI and the bridge nodes have to receive these MPI messages. Next, we explain our algorithm in detail in Section 3.1. In Section 3.2, we describe additional optimizations like node-level aggregation. In Section 3.3, we describe the modifications required for write calls when new bridge nodes are assigned. We outline some of the implementation details in Section 3.4.

\subsection{Bridge Node Search Algorithm}

In this section, we explicate our algorithm to search for new bridge nodes. Algorithm 1 illustrates the pseudo code for our algorithm. Lines 1-7 is the main skeleton of the algorithm, and there are two functions - BuildTree (lines 8-24) and Traverse (lines 25-38). The inputs to the algorithm are the set of bridge nodes $\mathcal{B}$ and the distance of each compute node to its default bridge node DefaultDistance. The algorithm outputs the new assignments of bridge nodes. The BuildTree function for constructing trees is detailed in Section 3.1.1. The Traverse function for traversing the trees for the reassignments is elaborated in Section 3.1.2.

\subsubsection{Tree Construction}

Each bridge node $\mathrm{b}$ constructs a tree Tree $[\mathrm{b}]$ rooted at itself. This tree is a connectivity tree wherein the edges represent the actual torus connectivity. An edge between two nodes in the tree represents the presence of a physical link between the nodes. A queue NodeQueue $[\mathrm{b}]$ is used to insert nodes into the tree by exploring the neighbors of the nodes in the queue. This queue helps in constructing the tree in breadth first order. The data structures Tree $[\mathrm{b}]$ and NodeQueue $[\mathrm{b}]$ are initialized to empty set in lines $2-3$. The first node inserted into the NodeQueue $[\mathrm{b}]$ is the bridge node $\mathrm{b}$ itself (line 4). BuildTree function is then invoked (line 5) to conditionally add nodes to the Tree[b]. First, we explore the neighbors of the bridge node followed by the neighbors of the neighbors of the bridge node i.e. the nodes which are 2 hops away from the bridge node and so on. This helps in discovering bridge nodes that are fewer hops away from a compute node than the default bridge node.

The tree is constructed level by level in breadth first order by examining the possibility of adding each neighbor $n$ of a node $x$ (lines 9-23), where $x$ is a node in the tree and the neighbors of node $x$ are currently being examined. We check if the path from $n$ to Root is a path in the current tree (line 10), where Root is the bridge node at which the tree is rooted. For this to happen, the message from $n$ to Root should be routed through $x$, where $x$ is the current node being expanded (see Figure 3). This path may or may not coincide with the edges in the tree (viz. $n \rightarrow x \rightarrow$ Root in Figure 3 ) due to the routing order. We explain next why this may be the case.

There is a pre-determined routing order in BG/Q and other similar systems with mesh and torus interconnects. BG/Q has a 5D torus with a static routing order determined by the 


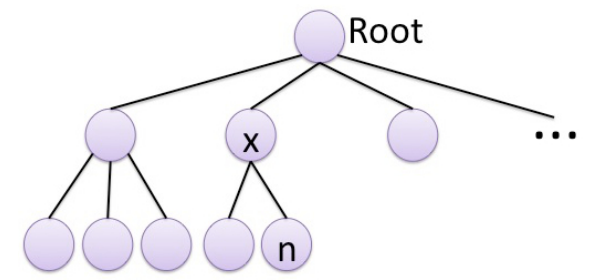

Figure 3: Tree rooted at a bridge node, children of a node in the tree are the neighbors of the node in the torus. $\mathrm{n}$ is a neighbor of $\mathrm{x}$. This tree is used to determine new bridge nodes for the compute nodes.

wiring of the nodes and specific to the partition(s) on which the job is running $[11,12,17]$. The routing order specifies the order of the dimensions along which the message is routed from the source node to the destination node. In our case, compute nodes are the source nodes and the bridge nodes are the destination nodes. The parent node of the current node may or may not be the node through which data is routed to the Root, depending on the routing order. For example, if $\mathrm{M}$ and $\mathrm{N}$ are the source and destination nodes respectively with coordinates $(3,2,2,1,1)$ and $(3,1,1,1,1)$, then the path from $\mathrm{M}$ to $\mathrm{N}$ would be $\mathrm{M}(3,2,2,1,1) \rightarrow$ $\mathrm{X}(3,1,2,1,1) \rightarrow \mathrm{N}(3,1,1,1,1)$ if the routing order is ABCDE. Whereas, if the routing order is ACBDE, the path would be $\mathrm{M}(3,2,2,1,1) \rightarrow \mathrm{Y}(3,2,1,1,1) \rightarrow \mathrm{N}(3,1,1,1,1)$. The message is routed via $\mathrm{B}$ dimension first in the case of $\mathrm{ABCDE}$ routing order, and the message is routed via $\mathrm{C}$ dimension first in the case of $\mathrm{ACBDE}$ routing order. The routing order can be found by reading the device control registers which are set at boot time.

In our algorithm, we check if the intermediate nodes on the path from $n$ to Root are nodes in the tree (line 10). This helps in reducing the network congestion by confining the I/O traffic within the paths in the tree. It is important that the newly added paths, due to reassignment of bridge nodes, do not lead to congestion and are able to reduce the network load imbalance as well. If this condition is satisfied, node $n$ is added as a child of node $x$ and node $x$ is assigned the parent of node $n$ (lines 11-12). This helps later in tree traversal when the actual assignment is done in function Traverse (lines 25-38). Further, the depth information of node $n$ is also updated in NewDistance[Root][n] to be the distance from the current bridge node Root (line 13). Hops(Root, n) returns the number of physical network links between Root and $n$. The node $n$ is enqueued in NodeQueue [b] for expansion later (line 14). We also update the node count of the tree in line 15. This is done so that we do not explore beyond MAXNODES (line 17). MAXNODES is typically set to total number of nodes/total number of bridge nodes which is the maximum number of compute nodes per bridge node. This also leads to similar count of nodes for every tree, and avoids load imbalance among the different bridge nodes.

If MAXNODES count of nodes is not reached, the BuildTree function will be recursively invoked for the front element of NodeQueue [b] (lines 17-19) otherwise the queue is emptied (line 21), signifying the end of the tree construction. Since we construct a tree in breadth first order, a tree consists of distinct nodes because the nodes are explored in the order of their distance from the root (bridge node). However a compute node may appear in one or more trees because it may be few hops away from multiple bridge nodes. Therefore, once all the trees are formed, we examine each tree rooted at a bridge node to decide the new assignment based on the distance from bridge node by invoking Traverse (line 7), which we outline in the next section. 


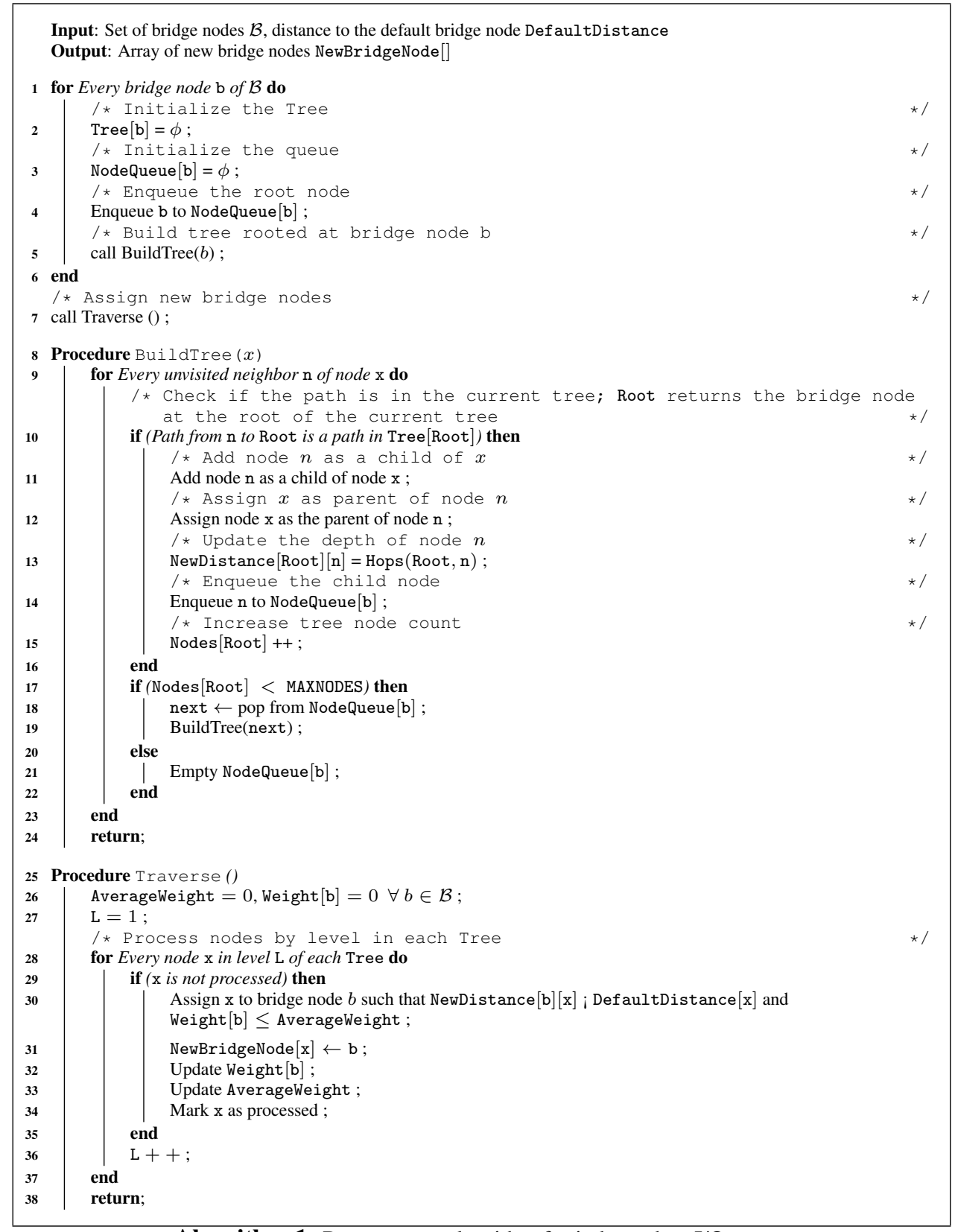

Algorithm 1: Route-aware algorithm for independent I/O.

\subsubsection{Tree Traversal}

The number of trees directly corresponds to the number of bridge nodes. In the function Traverse, each tree is traversed level by level, i.e. we do breadth first traversal across all the trees together. We illustrate this in Figure 4 which shows two trees rooted at Root $_{1}$ and 
$\operatorname{Root}_{2}$. It may be the case that a compute node $\mathrm{x}$ is two hops away from bridge node Root $_{1}$ while it is only one hop away from bridge node Root $_{2}$ as shown in the figure. Therefore, all the nodes in a particular level of all trees are examined before the nodes in the next level in any tree. This ensures that we examine first the compute nodes that are closer to any bridge node, and a compute node is assigned to its nearest bridge node.

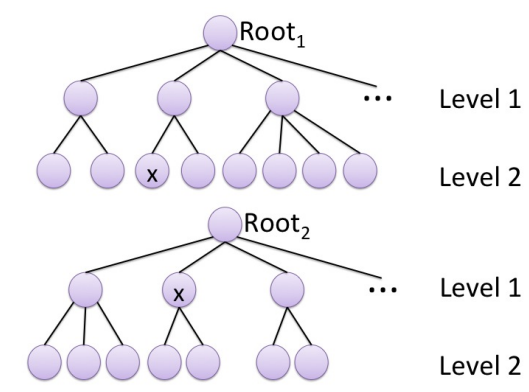

Figure 4: Collection of trees rooted at bridge nodes Root and Root $_{2}$. Node $\mathrm{x}$ may appear in multiple trees at different levels.

We start from level $\mathrm{L}=1$, whose nodes are one hop away from a bridge node (line 27). We examine every node in every level of each tree for certain condition satisfiability (line 28). Since a node may appear in multiple trees, we first check if the node is processed before or not (line 29). If the node, say $x$, is not processed before, it is assigned a new bridge node based on two conditions (line 30). The first condition is that the current depth of the node in the tree rooted at bridge node $b$, NewDistance $[\mathrm{b}][\mathrm{x}]$, is less than the distance from its default bridge node, DefaultDistance $[\mathrm{x}]$. The second condition is related to the number of new assignments for a bridge node, represented by Weight. We use the average number of new assignments, AverageWeight, to ensure that the number of new assignments (load) for all the bridge nodes is similar. They may not be exactly same due to integrality. AverageWeight and Weight of all bridge nodes are initialized to 0 in line 26 . The second condition requires Weight $[\mathrm{b}]$ to be less than or equal to the AverageWeight. Bridge node $b$ is assigned as the new bridge node for $x$ if the above two conditions are satisfied. This assignment is reflected in NewBridgeNode $[\mathrm{x}]$ (line 31). Weight $[\mathrm{b}]$ and AverageWeight are also updated (lines 32-33), and node $x$ is marked as processed (line 34). Level L is incremented (line 36) and the above steps are repeated for all levels.

\subsection{Intra-node aggregation}

In this section, we describe our aggregation schemes applied to the basic algorithm described in the previous section. The present era of multi-core systems witness high number of processing cores per node for better computing performance. For e.g., IBM Blue Gene/Q system has 16 cores per node, Intel Xeon Phi has 61 cores per node, etc. Thus, many applications commonly use multiple MPI ranks per node. Therefore, the read/write file system calls are also performed by multiple ranks within a node. This implies that there are multiple in-transit messages on the same route from a node to its bridge node, since different cores of a compute node are assigned to different cores on the same bridge node. Due to static routing between two nodes, all the messages from different ranks on the same node 
are routed on the same path in the BG/Q interconnect. This multiplies the interconnect congestion and the intra-node network contention, especially when there are higher number of MPI ranks per node. Further, larger message size leads to more traffic on the same links.

In our work, we aggregate messages per node in order to reduce the network traffic. For example, if there are 16 MPI ranks on the 16 cores of a BG/Q node, intra-node aggregation reduces the network traffic by 16 times. In our implementation, the MPI rank on $0^{\text {th }}$ core of a node aggregates the data from all the ranks in the node. After aggregation, the $0^{\text {th }}$ core sends the aggregated data either to the rank on $0^{\text {th }}$ core of the newly assigned bridge node or writes directly through its default bridge node on behalf of all ranks, if it is not assigned a new bridge node.

Message size plays an important role in the performance of our algorithm, with or without aggregation. Intra-node aggregation results in larger data transfers to the bridge nodes. Hence, aggregation at a single core improves performance for smaller message sizes. However, with increasing data size, one aggregator per node deteriorates performance primarily due to saturated network bandwidth. Additionally, one aggregator per node for larger message sizes results in increased overhead due to more packet transfers because of fixed network maximum transmission unit. Transfer time of aggregated data from one core of the compute node to one core of the bridge node is slightly higher than multiple simultaneous message transfers from all the cores. This is due to saturated network bandwidth with larger data size per rank and parallel message injection from multiple cores into the network injection FIFOs in BG/Q [11].

A BG/Q node has 16 GB DRAM per node, which is equally split between ranks on the same node. Therefore when 16 MPI ranks are used by an application, the available memory per core is $1 \mathrm{~GB}$. This restricts the number of aggregated messages that can be sent to a single rank on the bridge node, in the case of a single node-level aggregator. Thus, this limits Weight, the number of new assignments per bridge node as described in Section 3.1. We used Equation 1 to determine $\max W$ eight, the maximum value of Weight, where memAvail is the available memory per rank, messageSize is the data size per rank, and ppn is the number of MPI ranks per node. We determine memAvail at runtime, and use that to calculate maxWeight. We use this upper bound on Weight in Traverse function (Section 3.1.2). We modified Traverse function to ensure that Weight is always less than maxW eight for each bridge node, so that data from the newly assigned compute nodes fits in the memory of the bridge node.

$$
\text { maxWeight }=\text { memAvail } /(\text { messageSize } \times \text { ppn })
$$

There are several concurrent network flows on the same path if we do not perform any intra-node aggregation, whereas the performance drops if we use only one node-level aggregator for higher message sizes. Hence, we propose multiple aggregators per node which limits the number of concurrent network flows and also increases the threshold on Weight. We incorporate the number of aggregators as an additional parameter into Equation 1. We denote the number of aggregators per node by streams, and the modified upper limit on Weight is deduced using Equation 2. Thus, streams is a tunable parameter that provides the desired number of concurrent network flows between compute node and bridge node based on the data size.

$$
\text { maxWeight }=\text { memAvail } /\left(\text { messageSize } \times \frac{p p n}{\text { streams }}\right)
$$


The above intra-node aggregation approach is applicable to our modified optimized independent I/O, and is entirely different from MPI collective $\mathrm{I} / \mathrm{O}$ aggregators, which is based on file system accesses. In this work we assumed contiguous equi-sized data being written from different ranks in a node. However, the implementation is easily extensible to writing of data to random locations by transferring data size and location along with the data. In memory-intensive applications, there may not be sufficient memory at the bridge nodes, which are also the compute nodes. However, node-local storage and burst buffers on emerging systems $[1,3]$ will be useful in that case.

\subsection{Modified Write Algorithm}

In this section, we discuss the modifications required while invoking the write calls from the MPI ranks. We assume independent I/O here. The output of Algorithm 1 is NewBridgeNode[] which contains the new bridge node assignments. If a compute node is not assigned a new bridge node after the execution of Algorithm 1, MPI rank executing on the node writes its own data using MPI_File_iwrite_at. This results in the data being routed as system message to the default bridge node to be forwarded to one of the I/O nodes. If a compute node is assigned a new bridge node, the MPI rank in the node needs to send its data to this new bridge node. Therefore, the bridge nodes receive data and the compute nodes send data. We use non-blocking sends and receives for this purpose. Compute nodes send data using MPI_Isend and bridge nodes receive data using MPI_Irecv. Algorithm 2 illustrates this.

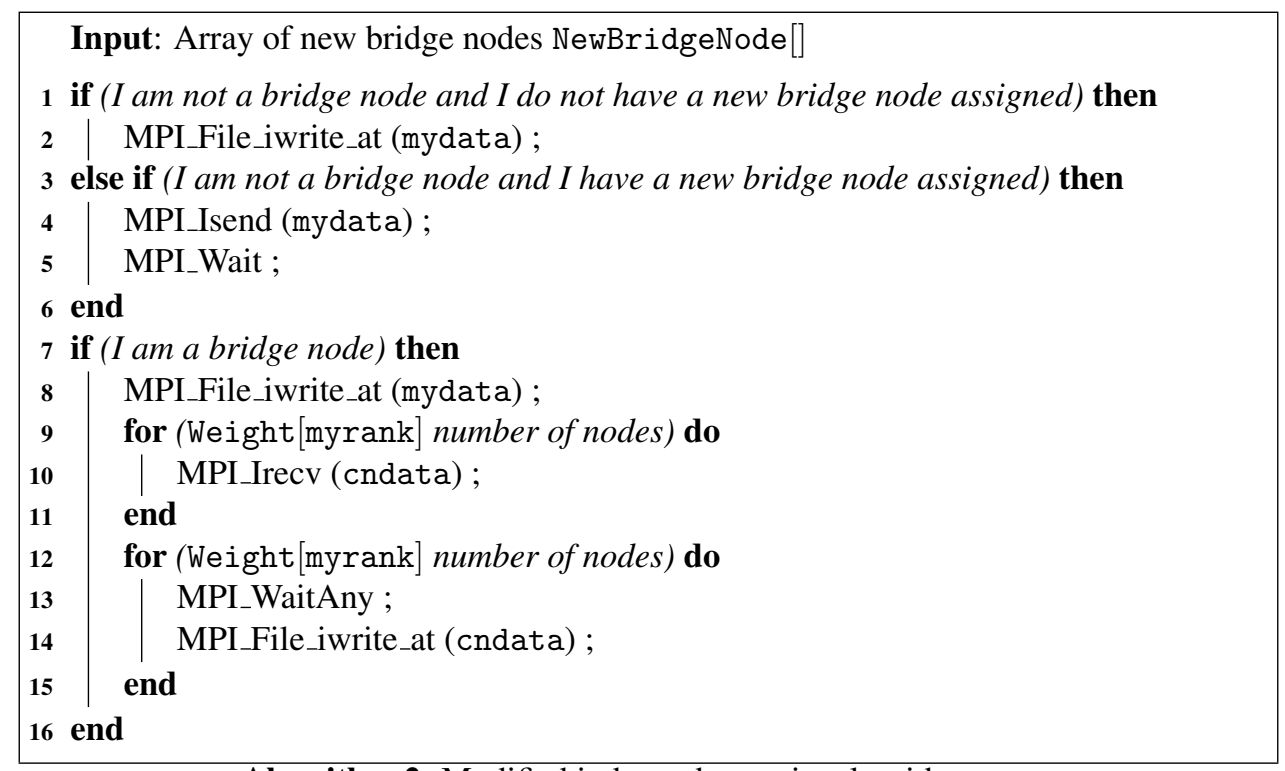

Algorithm 2: Modified independent write algorithm.

If a node is not a bridge node and is not assigned a new bridge node, then it writes its own data (lines 1-2). If a node is not a bridge node and is assigned a new bridge node, then it sends its data to this new bridge node (lines 3-6). The bridge nodes first write their own data (line 8). Then, they post non-blocking receives (lines 9-11) for the number of nodes 
that are assigned to them (represented by their Weight). The bridge nodes wait for any matching receive and write the data (cndata) received (lines 12-15). Since the distance to the compute nodes varies, it is beneficial to wait for any rank without any specific order. Algorithm 2 is executed by aggregator ranks if there is intra-node aggregation, and by every rank on a node, in the case of no aggregation.

\subsection{Implementation Details}

In this section, we report some of the implementation and hardware-specific details. The Blue Gene/Q Mira supercomputer, our evaluation platform [5], has two bridge nodes (and hence one I/O node) per 128 compute nodes. However, depending on the machine partition on which the job is run and the load on the I/O nodes, the default bridge nodes are configured such that they can lie anywhere in a BG/Q rack which comprises of 1024 compute nodes. Hence, for a job submitted on multiple racks, each rack of compute nodes can determine the new assignments simultaneously. Therefore, every rack executes Algorithm 1 in parallel. MPI_COMM_WORLD is split into sub-communicators for this purpose.

BG/Q has 4 hardware threads per core, 16 cores per node, and hence many applications use 16 MPI processes per node. All ranks in a node have the same bridge node. So, we execute Algorithm 1 per node and disseminate NewBridgeNode, if any, to all ranks in the node. It is worthwhile to note that multiple messages traverse on the same path to the bridge node from the multiple ranks in the node. This results in more network congestion in the already congested network (Figure 2). We reduce this congestion by combining data from all ranks and sending the combined message to the bridge node. In case of 1 aggregator per node, the aggregated data is sent from $0^{t h}$ core of the compute nodes to $0^{\text {th }}$ core of their new bridge nodes. Compute nodes without new bridge node assignment write through their default bridge node.

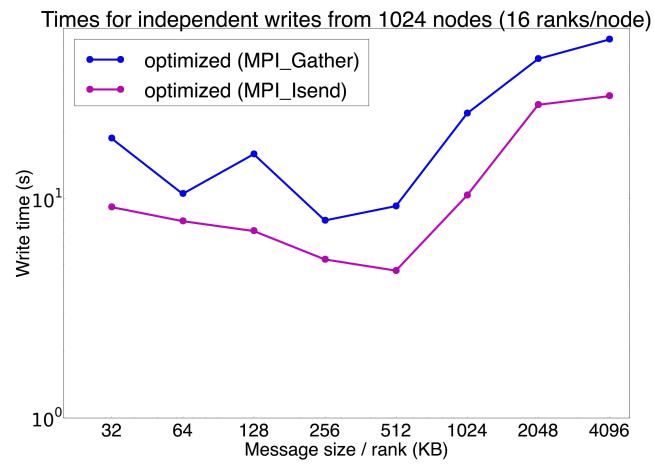

Figure 5: Time for writing 0.5 - 64 GB file from 1024 nodes, using two schemes for intra-node aggregation.

We used MPI_Gather for node-level aggregation in [26]. However, we found that pointto-point MPI sends and receives perform better for intra-node communications for all message sizes. Figure 5 shows time (y-axis) for writing 32 - $4096 \mathrm{~KB}$ (x-axis) per rank from 1024 nodes, 16 ranks/node, using MPI_Gather (blue curve) and MPI_Isend/Irecv (magenta curve) for intra-node aggregation on core 0 . We get $33-57 \%$ improvement with MPI_Isends because MPI_Gather uses a binomial tree algorithm, which incurs data copy overheads. The figure also shows latency-bound region below $512 \mathrm{~KB}$ message size, and bandwidth-bound 
communications above $512 \mathrm{~KB}$. In the case of multiple aggregators/node, they send aggregated data to respective cores on the bridge node. Here, we assume contiguous writes, and hence this results in coalesced access to the file system, which further leads to lower write times for independent writes. We show results for both with and without this optimization of combining messages per node, and with different number of aggregators per node.

The execution time of our algorithm to determine new bridge nodes is $\sim 0.1 \mathrm{sec}$ and it is a one-time cost at the start of the application. The space complexity is $\mathrm{O}(\mathrm{MN})$. $\mathrm{M}$ is the total number of bridge nodes and $\mathrm{N}$ is the total number of nodes per rack. $\mathrm{M}$ is a small constant fraction of $\mathrm{N}$. The space complexity is linear in the total number of nodes. We use 8-bit unsigned integers for most of the arrays used in Algorithm 1, thereby resulting in low memory overhead. Our optimized independent $\mathrm{I} / \mathrm{O}$ will incur lesser congestion and hence will also benefit MPI communication for applications using asynchronous I/O. Our optimizations will also benefit large-scale I/O-intensive applications. In Section 4, we show time to write data once. The savings in time will be higher for long-running large-scale simulations, which write terabytes of data per simulation run.

\section{Results and Discussion}

We performed all our experiments on Mira, the BG/Q at Argonne National Laboratory. Mira is a $10 \mathrm{PF}, 48$-rack machine. Each compute node has 16 cores and 16 GB RAM. Mira has a GPFS filesystem with $24 \mathrm{~PB}$ of capacity and a peak I/O bandwidth of $240 \mathrm{~GB} / \mathrm{s}$. Compute nodes are connected to $384 \mathrm{I} / \mathrm{O}$ nodes which connect to the GPFS file system over a QDR Infiniband network. We used 512 - 8192 nodes for our experiments. We submitted our jobs to the default queue. The batch scheduler in Mira selects the partition on which a job is run, which determines the I/O nodes placement [5]. Our results are averaged from 10 executions of each experiment; each job ran at non-overlapping times.

We present results for writing contiguous data from each rank, using MPI_File_iwrite_at for independent I/O and MPI_File_write_all for collective I/O. We study the impact of our algorithm on smaller and larger writes, ranging from $8 \mathrm{~KB}-4 \mathrm{MB}$ writes per MPI rank. The reported times for our algorithm include the time to write as well as aggregation times and MPI sends/receives for the new assignments. We used MPI_Isend for node-level aggregations. In the following subsections, we present results and discussion related to strong scaling, weak scaling, variation in number of ranks per node, variation in message size, variation in number of aggregators, reduction in number of hops, and metrics for network contention and load.

\subsection{Path to bridge node}

Figure 6 shows the path for I/O messages to bridge node 98 with our optimized algorithm for writing from 512 nodes with 1 rank per node. The graph was generated using Graphviz tool [4]. The edge width denotes the number of paths sharing the link, and the vertices denote the nodes of Mira. It shows the paths from 48 compute nodes which were assigned bridge node 98 based on our algorithm. We also show paths from the compute nodes whose default bridge node is 98 and were not assigned a new bridge node. Thus all the network flows into node 98 are depicted. Our algorithm leads to reassignments of bridge nodes to lessen network congestion and shorten distance to bridge nodes for I/O. Our approach reduces a total of 59 hops for compute nodes that are assigned to bridge node 98 . 


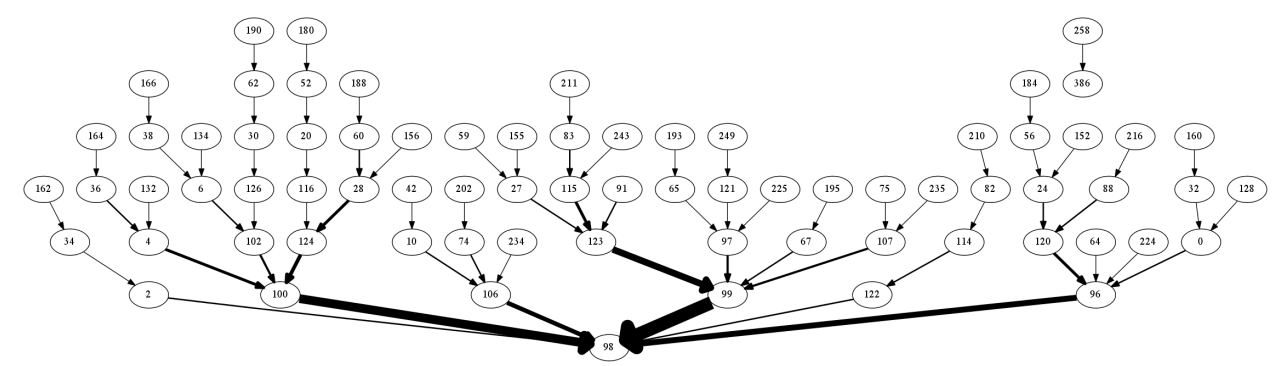

Figure 6: Path for I/O messages using our algorithm. Paths are shown from the compute nodes whose bridge node is node 98 . Node 98 is the newly assigned bridge node for 48 nodes, and default bridge node for the rest.

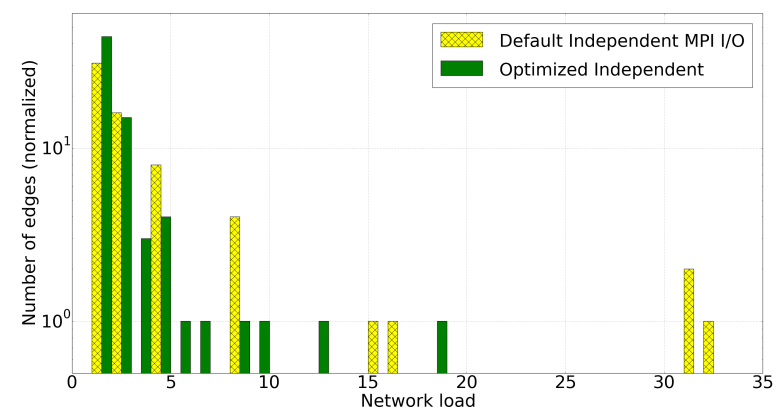

Figure 7: Histogram of network load for I/O paths from compute nodes to bridge node 98 in default approach and our approach.

Distance to bridge nodes is reduced for other compute nodes as well. In the above case, the average number of hops from compute nodes to their bridge nodes are 4.6 and 3.4 for the default independent I/O and our approach respectively.

We note that the network load is better balanced in our approach (Figure 6) than the default case (Figure 2). The maximum load on the interconnect links decreased from 32 in the default case to 18 with our approach. Figure 7 shows the histogram of load on the edges in Figures 2 and 6. The y-axis shows the normalized number of edges in default approach (patterned yellow bars) and our approach (green bars), x-axis shows network load. We observe that the number of edges with high load is much lesser with our algorithm. $48 \%$ edges have load 1 in the default approach whereas $60 \%$ edges in the network graph in Figure 6 have a load of 1, i.e. links without contention. Thus we reduce congestion by decreasing network load on many edges. The reduction in network load and number of hops to the bridge node is due to efficient bridge node assignment by our algorithm.

\subsection{Strong Scaling}

Figure 8 shows the time taken to write a 4 GB file using the default independent I/O, and our optimized independent I/O, with and without coalescing, strong scaling from 512 8192 nodes (4 ranks/node). At 512 nodes, each rank writes 2 MB data and at 8192 nodes, each rank writes $128 \mathrm{~KB}$ data. With increase in node count, the write time increases from 6.5 - 298.8 seconds for the default independent I/O and the time increases from $3.7-20.2$ seconds for our optimized independent I/O with 1 aggregator per node. The increase in time 
with higher process count is due to decrease in data size per rank which leads to higher lock contention.

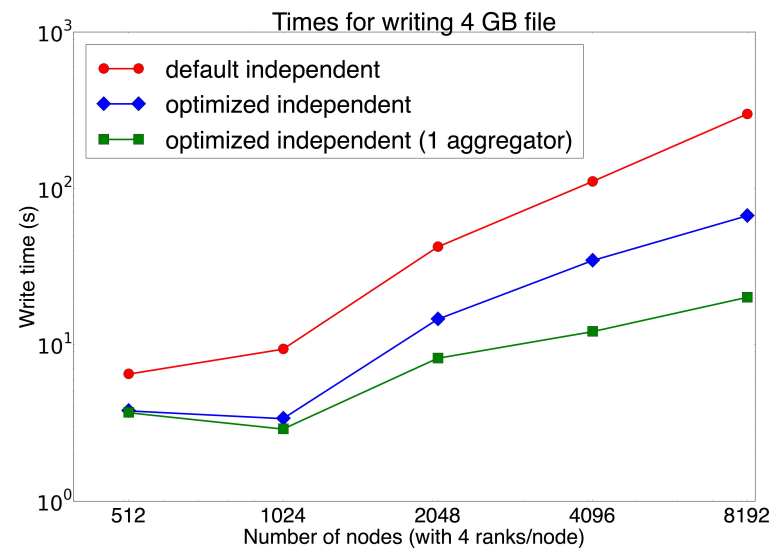

Figure 8: Strong scaling results on $512-8192$ nodes of Mira.

In the default case, the poor performance is not only due to more lock contention, but also due to longer hops to bridge node and higher network contention as was shown in Figure 2. Our optimized independent I/O, without aggregation, performs $4 \mathrm{x}$ better for this data size. This is because our algorithm finds closer bridge nodes and mitigates network contention. Furthermore, by combining data from all ranks in a node and then sending the data to the bridge nodes, our optimized independent $\mathrm{I} / \mathrm{O}$ with 1 aggregator leads to fewer I/O requests and hence performs $14 \times$ better at 8192 nodes than the default case. This clearly shows the importance of considering the system interconnect for I/O.

\subsection{Weak Scaling}

Figure 9 shows the write times for weak scaling on 512 - 8192 nodes of Mira. Figures 9(a) and 9(b) show the time taken using our optimized independent I/O both without and with coalescing/aggregating, and the default independent MPI-IO for $64 \mathrm{~KB}$ and 2 MB writes to the file system from each process, scaling from 512 - 4096 nodes with 4 ranks/node. Figures $9(\mathrm{c})$ and $9(\mathrm{~d})$ show the times for $64 \mathrm{~KB}$ and $2 \mathrm{MB}$ writes from each process, scaling from $512-4096$ nodes with 8 ranks/node. Thus, the maximum size of the file that was written to the GPFS filesystem is $64 \mathrm{~GB}$. We can note the difference in performance with our optimized algorithm due to assignments of new bridge nodes for some of the compute nodes.

For $64 \mathrm{~KB}$ writes, we observed an improvement from 9-20x with 4 ranks per node and $11-18 \mathrm{x}$ with 8 ranks per node. The coalescing of messages per node distinctly improves performance in the case of smaller writes due to high network bandwidth of the BG/Q 5D torus. Also, it results in reduced number of I/O requests to the file system. Our coalesced optimized algorithm (with 1 aggregator) for independent writes improved performance of 2 MB writes by 1.8x, 2x, 4x and 7x for 512, 1024, 2048 and 4096 nodes respectively, for both cases of 4 ranks/node and 8 ranks/node. With increasing number of processes, congestion in the multiple BG/Q racks, and therefore in the multiple I/O nodes, becomes more significant in the case of default independent MPI-IO. Our novel strategy to mitigate the bottleneck in 
the interconnect results in reduced write times. Thus our approach scales well on large core counts and large message sizes.

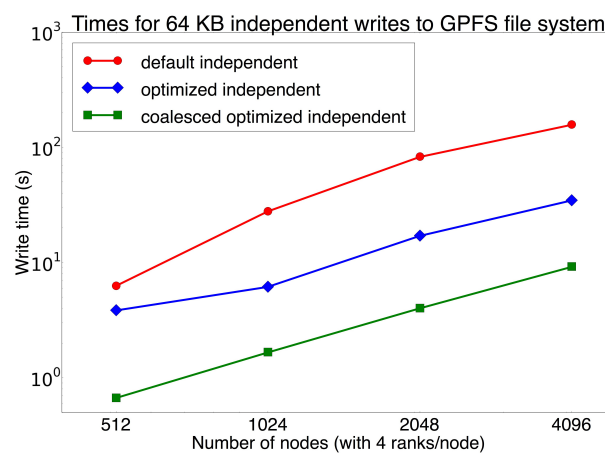

(a) $64 \mathrm{~KB}$ writes to GPFS (4 ranks/node).

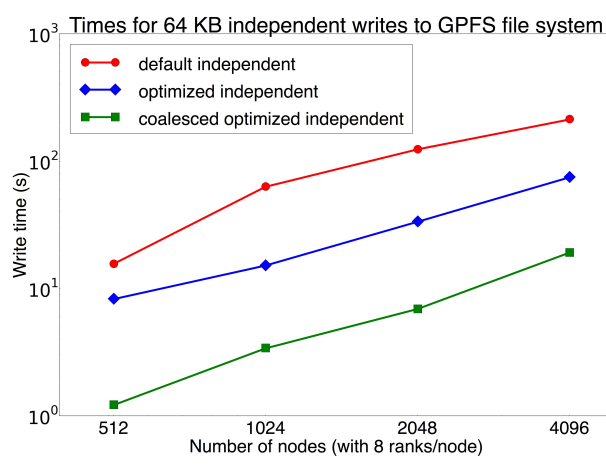

(c) $64 \mathrm{~KB}$ writes to GPFS (8 ranks/node).

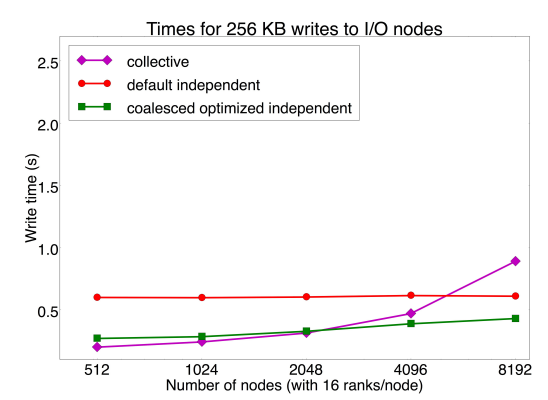

(e) $256 \mathrm{~KB}$ writes to $\mathrm{I} / \mathrm{O}$ nodes (16 ranks/node).

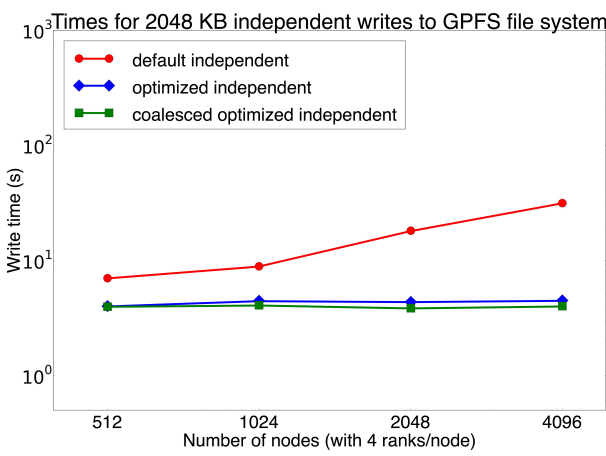

(b) $2 \mathrm{MB}$ writes to GPFS (4 ranks/node).

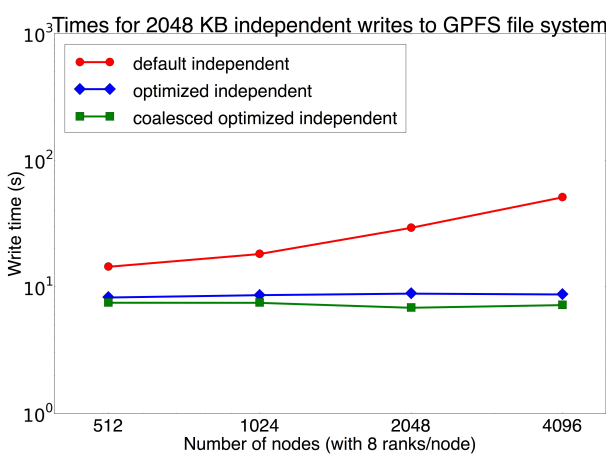

(d) 2 MB writes to GPFS (8 ranks/node).

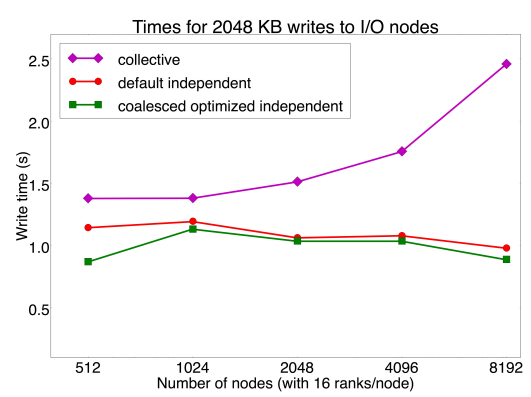

(f) $2 \mathrm{MB}$ writes to I/O nodes (16 ranks/node).

Figure 9: Weak scaling on $512-8192$ nodes, $4-16$ ranks/node and $64 \mathrm{~KB}-2 \mathrm{MB}$ writes per rank.

In Figures 9(e) and 9(f), we show the times for $256 \mathrm{~KB}$ and $2 \mathrm{MB}$ writes to the $\mathrm{I} / \mathrm{O}$ nodes from each MPI rank for jobs running on $512-8192$ nodes with 16 ranks/node. Thus the total file size varies from $16-256 \mathrm{~GB}$. We present this result to highlight the affect of congestion, if any, present in the interconnect while performing $\mathrm{I} / \mathrm{O}$. We observe that write 
times using collective I/O are more than both the default independent I/O and our optimized independent I/O. Our algorithm takes $3 \mathrm{x}$ less time to write $2 \mathrm{MB}$ as compared to collective I/O at 8192 nodes. In the case of independent I/O, messages travel from a compute node to the bridge node in one or more hops and then from bridge node to I/O node over the $11^{\text {th }}$ link. In the case of collective I/O, messages travel from a compute node to MPI aggregator nodes and then to the bridge node, and I/O node. In some cases, data is routed through many hops for both independent MPI-IO and collective MPI-IO. Through our route-aware algorithm of bridge node assignment for independent $\mathrm{I} / \mathrm{O}$, we reduce the number of hops and also confine each bridge node's traffic by using the connectivity tree for each bridge node.

Writing to the I/O node also emulates shipping of data to burst buffer nodes in future systems [23]. The results presented here elucidates the impact of our work. Though several optimizations are present in collective I/O to optimize file system accesses, independent I/O may perform better for writing to I/O nodes or burst buffer nodes. Furthermore, our routeaware optimized independent I/O algorithm outperforms the default independent I/O and collective I/O. Our results highlight the impact of network contention on the I/O times. Note that the time to write to $\mathrm{I} / \mathrm{O}$ nodes may not reflect the time to write to a file system which comprises of block devices and is also affected by file system lock contention [21, 22, 28].

\subsection{Multiple ranks per node}

I/O from multiple ranks per node results in more packets to travel on the same network path from a compute node to its bridge node due to static routing. This affects the performance of the default independent MPI I/O due to higher congestion as a result of highly loaded network links even with 1 rank per node. In this section we show the performance improvement with our optimizations when multiple ranks per node are used.

Figure 10 shows weak scaling results for time to write $128 \mathrm{~KB}$ and $1 \mathrm{MB}$ data per rank from 1024 nodes, varying from $1-16$ ranks per node. This results in $128 \mathrm{MB}$ file size for 1 rank per node and 2 GB file size with 16 ranks per node in the case of $128 \mathrm{~KB}$ writes (Figure 10(a)). For $1 \mathrm{MB}$ writes (Figure 10(b)), the total file size varies from $1 \mathrm{~GB}-$ 16 GB. We compare the default independent MPI I/O with our optimized independent I/O without node-level aggregation. We observe $2.2-4 \mathrm{x}$ improvement for these configurations. Node-level contention increases with increasing number of ranks per node. The write time increases from $7.17-36.09$ seconds in the case of default independent I/O for writing 128 $\mathrm{KB}$ from 1 - 16 ranks per node. Using our optimized independent I/O algorithm, the write times for $128 \mathrm{~KB}$ from 1 - 16 ranks per node increases from $2.09-11.55$ seconds. Thus, by devising a better message routing algorithm in the BG/Q interconnect, we are able to reduce the write time by more than $3 x$.

We also note the increase in write time with increasing ranks in the case of $1 \mathrm{MB}$ writes per rank (Figure 10(b)). This is because of increase in interconnect link contention with larger message sizes. However, larger message sizes also lead to lesser locking contention at the file system, and hence $1 \mathrm{MB}$ writes perform better than $128 \mathrm{~KB}$ writes. Also, smaller message sizes may result in latency-bound communications. Our optimized independent $\mathrm{I} / \mathrm{O}$ is able to reduce write times for $1 \mathrm{MB}$ messages from 4.39 to 1.96 seconds in the case of 1 rank per node, and from 41.29 to 7.34 seconds in the case of 16 ranks per node.

Figure 11 shows strong scaling results for time to write $1 \mathrm{~GB}$ file to the GPFS file system from 1024 nodes ( 1 rack) on Mira. Applications commonly use power of 2 number of ranks per node. Hence we show the results for $1-16$ ranks per node (x-axis) for default 


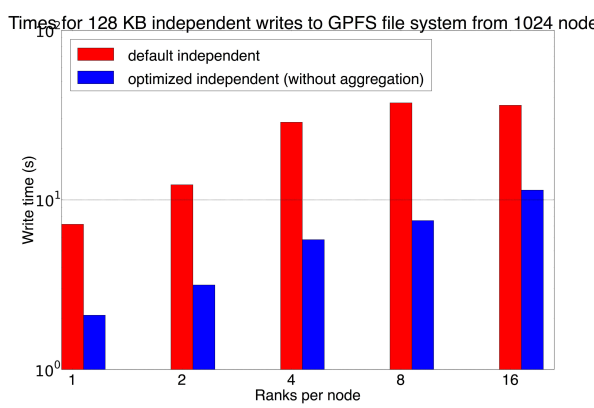

(a) $128 \mathrm{~KB}$ writes to GPFS.

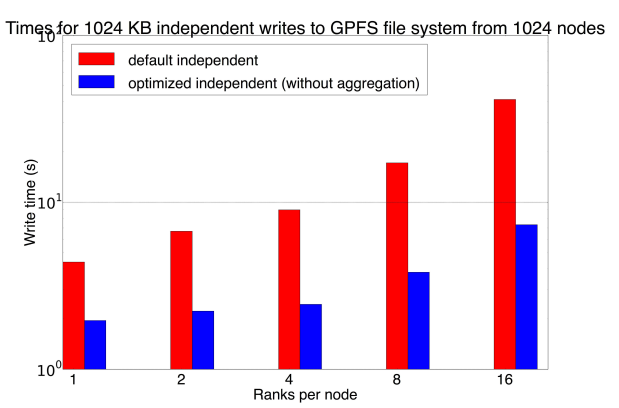

(b) $1024 \mathrm{~KB}$ writes to GPFS.

Figure 10: Weak scaling results on Mira, varying from 1024 - 16384 ranks on 1024 nodes, $128 \mathrm{~KB}-1$ MB writes per rank.

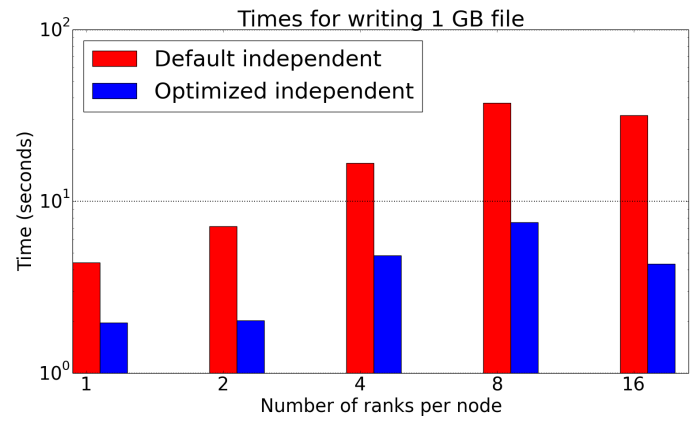

Figure 11: Time for writing 1 GB file from 1 - 16 ranks on 1024 nodes on Mira.

independent MPI I/O and optimized independent I/O (without aggregation). The y-axis shows the write time in seconds. The write data size per rank decreases with increasing number of ranks per node. Each rank writes $1024 \mathrm{~KB}$ at 1 rank/node (first point on X-axis), and each rank writes $64 \mathrm{~KB}$ at 16 ranks per node (last data point on $\mathrm{x}$-axis). The data written by each rank decreases when more number of ranks are used. However, to write the same file size ( $1 \mathrm{~GB})$, the time taken increases from 4.39 - 31.73 seconds in the default case. We observed 2.2x, 3.5x, 3.5x, 4.9x and 7.3x improvement at 1, 2, 4, 8 and 16 ranks respectively with our approach, without intra-node aggregation. One of the main reasons for increase in time with increase in number of ranks (or decrease in data size) is higher locking contention in the file system due to smaller writes.

We reassign bridge nodes based on the distance to bridge nodes, network connectivity graph and the BG/Q routing algorithm, hence our algorithm improves performance over the default case for varying ranks per node. Our algorithm reduces network congestion and hence gains are higher for higher ranks/node. In the case of default MPI independent I/O, the congestion is higher with more number of ranks per node because more packets are routed on the same links. Also, longer hops to the bridge nodes result in higher latency for multiple messages from multiple ranks in a node, even though the data size is smaller at higher ranks. In the case of our optimized algorithm also, more number of packets are routed along the same path when multiple ranks/node write data simultaneously. However, 


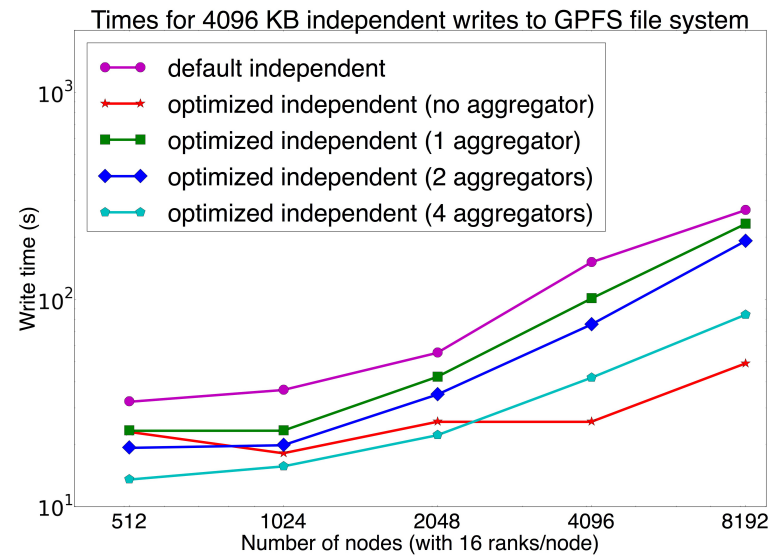

Figure 12: File write times for default MPI independent I/O and our approach, from 512 - 8192 nodes on Mira with 16 ranks per node for $4 \mathrm{MB}$ writes per rank.

due to better network utilization and shorter hops, our algorithm reduces the write times.

As seen in Figure 11, at 16 ranks per node with each rank writing $64 \mathrm{~KB}$, we are able to reduce write times by 27.4 seconds for a single write step. This implies around 1 hour reduction in total I/O time for a large-scale simulation run of 10000 time steps, output frequency being every 50 time steps. The savings in time can be more significant for large-scale cosmological simulations like HACC [18], which uses independent I/O as one of the I/O modes and writes terabytes of data per simulation run. In our case, using multiple aggregators per node further reduces I/O times in most cases, as discussed in the next section.

\subsection{Multiple aggregators per node}

Figure 12 shows weak scaling results for writing $4 \mathrm{MB}$ data/rank from $512-8192$ nodes (x-axis) on Mira with 16 ranks/node. The times on y-axis are shown for the default MPI independent I/O and our approach using $0,1,2$, and 4 aggregators/node. The times include aggregation time, point-to-point communication times and file write times. The total file size written to the file system varies from $32-512$ GB. However, the data size per network flow varies for each configuration (number of aggregators). In the case of $0 \mathrm{ag}$ gregators/node, i.e. without intra-node aggregation, all 16 ranks of a node write their data, either through the default bridge node or through newly assigned bridge node. The number of aggregators determine the number of concurrent network flows originating from a node. There are 16 concurrent network flows (from 16 ranks) along the same path without aggregating data ( 0 aggregators). Node-level aggregation results in reduced number of concurrent network flows on the same path. Therefore the size of data on a network flow originating from each node depends on whether aggregation is used and the number of aggregators.

We got a maximum improvement of $5.8 \mathrm{x}$ with our approach over the default independent MPI I/O for this data size. New assignment involves sending data from compute nodes and receiving data at bridge nodes. There are 8, 16, 32, 64 and 128 bridge nodes for a BG/Q partition of 512, 1024, 2048, 4096 and 8192 nodes respectively, i.e. the ratio of number of bridge nodes to number of compute nodes is 1:64. Hence, larger the data size per node, fewer is the number of new assignments per bridge node. Also, fewer the number 
Table 1: Number of nodes with new bridge node assignments.

\begin{tabular}{|l||l|l|l|l|}
\hline \#Nodes & 1 aggregator & 2 aggregators & 4 aggregators & No aggregation \\
\hline 512 & 48 & 104 & 216 & 384 \\
\hline 1024 & 96 & 208 & 432 & 576 \\
\hline 2048 & 192 & 416 & 864 & 1888 \\
\hline 4096 & 384 & 832 & 1728 & 3200 \\
\hline
\end{tabular}

of aggregators per node, larger the data size sent from the aggregator cores on compute nodes to the corresponding core on the bridge node. Thus this limits the number of new assignments due to limited memory available on each rank of the bridge nodes as described in Section 3.2.

Table 1 shows the number of compute nodes which were assigned new bridge nodes for each data point in Figure 12. The first column in the table shows the number of nodes. We observe that with 1 aggregator, the write times are higher than with 2 aggregators, and performance with 2 aggregators is worse than 4 aggregators for all node counts in Figure 12. Our optimizations with 4 aggregators improve performance by $33-59 \%$ over 1 aggregator, and $21-45 \%$ over 2 aggregators for this data size. This is because more number of new assignments is possible with 4 aggregators as shown in column 4 of Table 1 . The data size per network flow from aggregator core(s) in a node is 64,32 , and $16 \mathrm{MB}$ with 1,2 , and 4 aggregators per node. $16 \mathrm{MB}$ data is sent from each of the 4 aggregator cores (cores $0,4,8$, 12) on a node for 4 aggregators per node. Thus, $16 \mathrm{MB}$ data is received at the corresponding bridge node cores (cores $0,4,8,12$ ) from corresponding aggregator cores on all the newly assigned compute nodes. However, for 1 aggregator per node, $64 \mathrm{MB}$ data is received at core 0 of each bridge node from each of the newly assigned compute nodes. Thus, with 1 aggregator (shown in column 2), 48 out of 512 nodes have new bridge nodes assigned according to our algorithm, whereas with 4 aggregators, 216 out of 512 nodes have new bridge nodes which implies lower interconnect congestion in the 512-node partition.

We also note that the performance of 4 aggregators is better than 0 aggregators from 512 -2048 nodes. Without aggregation, each rank sends 4 MB data to its assigned bridge node, which causes node-level contention because all 16 ranks send data using the same network interface. It also increases latency per hop due to 16 different network flows on the same path, as compared to 4 network flows with 4 aggregators. The performance improves by aggregating data because of high-bandwidth interconnect. Additionally, larger data writes (16 MB) with 4 aggregators cause lesser file locking contention at the file system, since the default GPFS block size is $8 \mathrm{MB}$. However, at 4096 nodes, while writing to a $256 \mathrm{~GB}$ file, the best performance is achieved without intra-node aggregation. Few possible reasons could be high number of new assignments (3200) with 0 aggregators and the availability of more number of bridge nodes at 4096 nodes, and hence more number of I/O nodes. We plan to investigate this further in future.

\subsection{Message size variation}

Table 2 shows the percentage decrease in write times with our optimized independent I/O, without aggregation and with 2 aggregators per node, on 512 and 4096 nodes on Mira (16 ranks per node). The write times for our approach are also specified in parenthesis. We observe $60-95 \%$ improvement for $128-1024 \mathrm{~KB}$ writes per rank. The total file size varies 
from $1-8 \mathrm{~GB}$ at 512 nodes, and $8-64$ GB at 4096 nodes. For almost all cases, sending aggregated data from 2 cores in a node gives better performance than sending data from each of the 16 cores in a node. This is because of lower node-level contention and better interconnect bandwidth utilization. Thus we are able to achieve $10.5 \mathrm{~GB} / \mathrm{s}$ write bandwidth at 4096 nodes writing $64 \mathrm{~GB}$ file, as compared to $0.7 \mathrm{~GB} / \mathrm{s}$ with default MPI independent $\mathrm{I} / \mathrm{O}$. The results show that our approach works well for different message sizes and at scale.

Table 2: Percentage improvement (decrease in write times) with 128 - 1024 KB data size per rank from 512 and 4096 nodes on Mira (16 ranks per node).

\begin{tabular}{|l||l|l|l|l|}
\hline Message size (KB) & 128 & 256 & 512 & 1024 \\
\hline \multicolumn{5}{|c|}{512 nodes } \\
\hline Default time (s) & 31.31 & 32.07 & 31.72 & 31.49 \\
\hline No aggregation & $79.9 \%(6.29 \mathrm{~s})$ & $85.6 \%(4.62 \mathrm{~s})$ & $86.8 \%(4.20 \mathrm{~s})$ & $77.3 \%(7.16 \mathrm{~s})$ \\
\hline 2 aggregators & $94.8 \%(1.63 \mathrm{~s})$ & $93.2 \%(2.18 \mathrm{~s})$ & $91.4 \%(2.73 \mathrm{~s})$ & $83.1 \%(5.32 \mathrm{~s})$ \\
\hline \multicolumn{5}{|l|}{4096 nodes } \\
\hline Default time (s) & 109.92 & 95.78 & 83.72 & 92.47 \\
\hline No aggregation & $61.9 \%(41.87 \mathrm{~s})$ & $75.0 \%(23.94 \mathrm{~s})$ & $94.3 \%(4.76 \mathrm{~s})$ & $91.7 \%(7.68 \mathrm{~s})$ \\
\hline 2 aggregators & $79.9 \%(22.03 \mathrm{~s})$ & $82.6 \%(16.63 \mathrm{~s})$ & $93.6 \%(5.39 \mathrm{~s})$ & $93.4 \%(6.08 \mathrm{~s})$ \\
\hline
\end{tabular}

\subsection{Network Contention}

In this section, we present results regarding the interconnect contention using the BG/Q network counters. Table 3 shows the average number of messages routed through a bridge node while writing $64 \mathrm{~KB}$ and $2 \mathrm{MB}$ from 2048 nodes. Columns 2 and 3 show results for default and optimized independent I/O respectively with 4 ranks/node. Columns 4 and 5 show results for default and optimized independent I/O respectively with 8 ranks/node. Note that the average number of messages routed through a bridge node using the default independent I/O is 1.4 times higher in all cases. This implies higher load on the bridge nodes because in the default case, the bridge nodes may also be the intermediate nodes through which messages are routed to other bridge nodes. Our tree construction algorithm does not assign any bridge node as an intermediate node in the tree. Our algorithm assigns closer bridge nodes without overloading any bridge node with the new assignments. Additionally we select nodes by considering the routing order to build the connectivity tree, such that the entire network is not congested due to traffic arising from the new assignments. Thus contention at bridge nodes is less in our case.

Table 3: Average number of messages routed through a bridge node while writing $64 \mathrm{~KB}$ and $2 \mathrm{MB}$ from 2048 nodes with 4 ranks/node (columns $2-3$ ) and 8 ranks/node (columns $4-5$ ).

\begin{tabular}{||l||l|l||l|l||}
\hline \multirow{2}{*}{ Data size } & \multicolumn{2}{|c|}{4 ranks/node } & \multicolumn{2}{c||}{8 ranks/node } \\
& Default & Optimized & Default & Optimized \\
\hline \hline $64 \mathrm{~KB}$ & 12.7 & 8.5 & 25.6 & 17.5 \\
\hline $2 \mathrm{MB}$ & 407.7 & 289.0 & 815.5 & 581.5 \\
\hline \hline
\end{tabular}

Table 4 shows the normalized standard deviation in the number of messages transmitted from bridge nodes. This gives an indication of imbalance in network load at the bridge 
nodes, through which data from all compute nodes reach the I/O nodes. Rows $1-3$ show the deviation for writing $64 \mathrm{~KB}$ from each rank when running on 512, 1024 and 2048 nodes. Rows $4-6$ show the deviation for writing 2048 MB from each rank when running on 512, 1024 and 2048 nodes. We observe an order of magnitude difference between the results for the default and optimized independent I/O. For the case of default independent I/O, many compute nodes are assigned a bridge node which is not the nearest one. This leads to lot of network traffic at the bridge nodes since they route traffic to compute nodes in addition to sending data to the I/O nodes. This is more significant for larger messages since transmitting larger messages implies transmission of more packets depending on the network maximum transmission unit. Lesser variation in the number of messages transmitted from bridge nodes implies more balanced load for our optimized independent I/O.

Table 4: Standard deviation in number of messages sent from bridge nodes while writing $64 \mathrm{~KB}$ (first three rows) and 2 MB (last three rows) data from 512, 1024 and 2048 nodes with 1 rank/node.

\begin{tabular}{||l|l|l|l|l||}
\hline Data size & \#Nodes & Default & $\begin{array}{l}\text { Optimized (No } \\
\text { aggregation) }\end{array}$ & $\begin{array}{l}\text { Optimized (1 } \\
\text { aggregator) }\end{array}$ \\
\hline \multirow{3}{*}{$64 \mathrm{~KB}$} & 512 & 0.09 & 0.02 & 0.02 \\
& 1024 & 0.05 & 0.01 & 0.01 \\
& 2048 & 0.08 & 0.002 & 0.007 \\
\hline \multirow{3}{*}{$2 \mathrm{MB}$} & 512 & 0.07 & 0.02 & 0.03 \\
& 1024 & 0.11 & 0.01 & 0.01 \\
& 2048 & 0.11 & 0.002 & 0.004 \\
\hline
\end{tabular}

\section{Conclusions}

Improved $\mathrm{I} / \mathrm{O}$ performance results in improved performance of $\mathrm{I} / \mathrm{O}$-intensive applications. In this work, we have shown that better routing in the interconnect affects I/O performance. We improved independent I/O performance on Blue Gene/Q by modifying the bridge node assignments in a route-aware approach. We show that it is important to consider the route of I/O messages within the interconnect before they reach the I/O nodes or the file system. We demonstrate at scale that by mitigating the network congestion, one can significantly decrease the I/O times. Our algorithm gives $60 \%$ better performance on average than the default independent I/O for writing to the file system from $512-131072$ processes on Mira. We observed a $20 \%$ average performance improvement over collective I/O while writing $128 \mathrm{~KB}-8 \mathrm{MB}$ per rank to the I/O nodes from $8192-131072$ processes. The default independent MPI I/O routes $1.4 \times$ more I/O messages through the bridge nodes. We also presented intra-node aggregation strategy to further improve performance. Our optimizations with more number of aggregators can improve performance by up to $4 \times$ as compared to using 1 aggregator per node. With intra-node aggregation, we achieved 10.5 $\mathrm{GB} / \mathrm{s}$ write bandwidth at $64 \mathrm{~K}$ ranks writing a $64 \mathrm{~GB}$ file, as compared to $0.7 \mathrm{~GB} / \mathrm{s}$ with default MPI independent I/O.

We have demonstrated our approach on the Blue Gene/Q architecture. While we focussed on Blue Gene/Q's 5D torus interconnect and its I/O forwarding layer, we believe our algorithm will be applicable on other systems as well. The Cray supercomputer Edison, which has the dragonfly interconnect and Lustre file system, uses LNET nodes for I/O 
forwarding. This mechanism is similar to the BG/Q I/O nodes, as considered in this work. In future, we plan to experiment on Cray systems. Most of the adaptive routing decisions in HPC interconnects (viz. dragonfly, 5D torus, etc.) are based on the local inflow and outflow of traffic from a node since it is costly to implement globally adaptive routing. Our algorithm routes traffic using the global knowledge about the interconnect topology and the I/O paths from a compute node to its bridge node. While the current routing for I/O on BG/Q does not use adaptive routing, however it can be complementary to our approach. Our work will also be useful in future systems equipped with burst buffers which may be connected to few gateway nodes in the system, similar to the current I/O node architecture. Our algorithm will improve the read times as well, since the read times are also affected by the interconnect contention. In future, we plan to release our work as an application-level library.

\section{Acknowledgments}

This research has been funded in part and used resources of the Argonne Leadership Computing Facility at Argonne National Laboratory, which is supported by the Office of Science of the U.S. Department of Energy under contract DE-AC02-06CH11357. This work was supported in part by the DOE Office of Science, Advanced Scientific Computing Research, under award number 57L38, 57L32, 57K07 and 57K50. The authors would also like to thank Philip Heidelberger and Adam Scovel for their help with BG/Q routing algorithm and I/O node configurations.

[1] Aurora. http://aurora.alcf.anl.gov.

[2] CORAL benchmarks. https://asc.llnl.gov/CORAL-benchmarks/.

[3] Cori. http://www.nersc.gov/users/computational-systems/ cori.

[4] Graphviz. http://www.graphviz.org.

[5] Mira. http://www.alcf.anl.gov/user-guides/mira-cetus-vesta.

[6] P. Balaji and et al. Mapping communication layouts to network hardware characteristics on massive-scale blue gene systems. Computer Science - Research and Development, 26(3-4):247-256, 2011.

[7] A. Bhatele and et al. There Goes the Neighborhood: Performance Degradation Due to Nearby Jobs. In Proc. of the International Conference on High Performance Computing, Networking, Storage and Analysis, SC '13, 2013.

[8] P. Carns and et al. 24/7 Characterization of Petascale I/O Workloads. In Proc. of the First Workshop on Interfaces and Abstractions for Scientific Data Storage, 2009.

[9] P. Carns and et al. Understanding and improving computational science storage access through continuous characterization. Trans. Storage, 7(3):8:1-8:26, 2011.

[10] P. Carns and et al. Production I/O Characterization on the Cray XE6. In CUG2013, 2013. 
[11] D. Chen and et al. The IBM Blue Gene/Q Interconnection Network and Message Unit. In Proceedings of International Conference for High Performance Computing, Networking, Storage and Analysis, SC '11, 2011.

[12] D. Chen and et al. Looking Under the Hood of the IBM Blue Gene/Q Network. In Proceedings of the International Conference on High Performance Computing, Networking, Storage and Analysis, SC '12, 2012.

[13] J. H. Chen and et al. Terascale direct numerical simulations of turbulent combustion using S3D. Computational Science \& Discovery, 2(1), 2009.

[14] Y. Chen, X.-H. Sun, R. Thakur, P. Roth, and W. Gropp. LACIO: A New Collective I/O Strategy for Parallel I/O Systems. In Parallel Distributed Processing Symposium (IPDPS), 2011 IEEE International, 2011.

[15] D. A. Dillow and et al. Enhancing I/O Throughput via Efficient Routing and Placement for Large-scale Parallel File Systems. In Proc. of 30th IEEE International Performance Computing and Communications Conference, 2011.

[16] S. El Sayed and et al. Using GPFS to Manage NVRAM-Based Storage Cache. In J. Kunkel, T. Ludwig, and H. Meuer, editors, Supercomputing, volume 7905 of Lecture Notes in Computer Science, pages 435-446. Springer Berlin Heidelberg, 2013.

[17] M. Gilge. IBM System Blue Gene Solution: Blue Gene/Q Application Development. IBM Redbooks, June 2013.

[18] S. Habib, V. Morozov, N. Frontiere, H. Finkel, A. Pope, and K. Heitmann. Hacc: Extreme scaling and performance across diverse architectures. In Proceedings of the International Conference on High Performance Computing, Networking, Storage and Analysis, SC'13, pages 6:1-6:10, 2013.

[19] R. Haring and et al. The IBM Blue Gene/Q Compute Chip. Micro, IEEE, 32(2):48-60, 2012.

[20] S. Kumar, Y. Sabharwal, R. Garg, and P. Heidelberger. Optimization of All-to-All Communication on the Blue Gene/L Supercomputer. In Parallel Processing, 2008. ICPP '08. 37th International Conference on, pages 320-329, 2008.

[21] R. Latham and et al. A case study for scientific I/O: improving the FLASH astrophysics code. Computational Science \& Discovery, 5(1), 2012.

[22] W.-k. Liao and et al. Using MPI File Caching to Improve Parallel Write Performance for Large-scale Scientific Applications. In Proceedings of International Conference for High Performance Computing, Networking, Storage and Analysis, SC '07, 2007.

[23] N. Liu and et al. On the role of burst buffers in leadership-class storage systems. In IEEE 28th Symposium on Mass Storage Systems and Technologies, April 2012.

[24] J. Lofstead, F. Zheng, S. Klasky, and K. Schwan. Adaptable, metadata rich IO methods for portable high performance IO. In Parallel Distributed Processing, 2009. IPDPS 2009. IEEE International Symposium on, 2009. 
[25] P. Malakar and V. Vishwanath. Hierarchical Read-write Optimizations for Scientific Applications with Multi-variable Structured Datasets. In 12th IFIP International Conference on Network and Parallel Computing (NPC 2015). 2015.

[26] P. Malakar and V. Vishwanath. Route-aware Independent MPI I/O on the Blue Gene/Q. In Proceedings of the 2015 International Workshop on Data-Intensive Scalable Computing Systems, DISCS '15, pages 5:1-5:8, 2015.

[27] R. Rajachandrasekar, J. Jaswani, H. Subramoni, and D. Panda. Minimizing Network Contention in InfiniBand Clusters with a QoS-Aware Data-Staging Framework. In Cluster Computing (CLUSTER), 2012 IEEE International Conference on, pages 329336, 2012.

[28] F. Schmuck and R. Haskin. GPFS: A Shared-Disk File System for Large Computing Clusters. In Proceedings of the 1st USENIX Conference on File and Storage Technologies, FAST'02, 2002.

[29] P. Schwan. Lustre, Building a file system for 1000 node clusters. In Proceedings of Linux Symposium, 2003.

[30] R. Thakur, W. Gropp, and E. Lusk. Data Sieving and Collective I/O in ROMIO. In Proceedings of the The 7th Symposium on the Frontiers of Massively Parallel Computation, FRONTIERS '99, 1999.

[31] A. Uselton and et al. Parallel I/O performance: From events to ensembles. In Parallel Distributed Processing (IPDPS), 2010 IEEE International Symposium on, 2010.

[32] Z. Wang and et al. Iteration Based Collective I/O Strategy for Parallel I/O Systems. In Cluster, Cloud and Grid Computing, 14th IEEE/ACM International Symposium on, May 2014.

[33] H. Yu and et al. High performance file I/O for the Blue Gene/L supercomputer. In Twelfth International Symposium on High-Performance Computer Architecture., 2006. 